\title{
What perspectives underlie 'researcher identity'? A review of two decades of empirical studies
}

\author{
Montserrat Castelló ${ }^{1,2}$ (D) $\cdot$ Lynn McAlpine ${ }^{3,4}$ - Anna Sala-Bubaré ${ }^{1} \cdot$ Kelsey Inouye $^{3}$. \\ Isabelle Skakni ${ }^{5}$
}

Published online: 23 June 2020

(C) The Author(s) 2020

\begin{abstract}
Over the past two decades, identity has emerged as a concept framing studies of early career researcher experience. Yet, identity is an amorphous concept, understood and used in a range of ways. This systematic review aimed to unpack the underpinnings of the notion of researcher identity. The final sample consisted of 38 empirical articles published in peer-reviewed journals in the last 20 years. Analyses focused on (a) identifying the dimensions used to define researcher identity, and (b) characterising the meta-theoriesthe underlying assumptions of the research - in relation to these dimensions. We identified four different stances towards researcher identity (clusters), based on variation on the identity dimensions in relation to the meta-theories. We characterised these as (1) transitioning among identities, (2) balancing identity continuity and change, (3) personal identity development through time and (4) personal and stable identity. These stances incorporate thought-provoking nuances and complex conceptualisations of the notion of researcher identity, for instance, that meta-theory was insufficient to characterise researcher identity stance. The contribution of the study is first to be able to differentiate four characterizations of researcher identity-important given that many studies had not clearly expressed a stance. The second is the potential of the four dimensions to help characterise identity, in past as well as future research - thus a useful tool for those working in this area. Many questions remain, but perhaps the biggest is to what extent and under what conditions is identity a productive notion for understanding early career researcher experience?
\end{abstract}

Keywords Researcher identity · Meta-theories $\cdot$ Identity dimensions $\cdot$ Theories $\cdot$ Identity

Electronic supplementary material The online version of this article (https://doi.org/10.1007/s10734-02000557-8) contains supplementary material, which is available to authorized users.

Montserrat Castelló

montserratcb@blanquerna.url.edu

Extended author information available on the last page of the article 


\section{Introduction}

Today, in many parts of our lives, 'identity' is a recurrent concept, that although frequently contested, is often used to frame human activity in various domains, such as digital, social or genre identity. Increasingly, the concept of 'identity' has been invoked over the past two decades in the higher education literature, including that of early career researchers $(\mathrm{PhD}$ and post- $\mathrm{PhD}$ researchers). Studies frequently claim identity as a central aspect of early career researcher development and the extent to which they manage to develop a sound identity as researchers is crucial to their professional success (Alvesson, Ashcraft and Thomas 2008; Castelló et al. 2015). However, we ${ }^{1}$ argue the representations of identity in such empirical studies are rarely challenged, instead being largely presented as a start- or end-point for examining early researcher career experiences. For instance, studies on doctoral students often include reference to Green's (2005) notion of the $\mathrm{PhD}$ as identity work in their introductions, but do not usually define what it means in the context of the study (Baker and Lattuca 2010).

In other words, identity is not a straightforward notion and has multiple embedded meanings. Still, it can be a useful device since it is 'a tool to think about sameness and difference both in terms of individual continuity and change over time and social categorization or group affiliation' (Hammack 2015, p. 11). So, if 'identity' is to be useful and meaningful as a concept that can advance research into early career researcher experience, we need to fully understand the assumptions behind the view of identity taken in any particular study, since such assumptions will drive the research and colour the resulting interpretations (Mac Naughton, Rolfe and Siraj-Blatchford 2001). As several authors have argued, it is not possible - nor appropriate - to provide a single, overarching definition of identity. Rather, we need to start with the theoretical underpinnings underlying each particular definition or study (Hall 1992; Strauss 2017; Hammack 2015). In this systematic review (Kennedy 2007), we critically analysed the empirical literature on researcher identity in order to 'unpack' the varied theoretical underpinnings of identity — and concurrently, our own respective definitions and assumptions (Grant and Booth 2009).

Analysing the underlying dimensions of researcher identity is especially necessary today. In the last decade, institutions, stakeholders and policies around the world have called for a new researcher profile able to develop responsible research and innovation to make possible science with and for society (SWAFS, Horizon 2020). To understand what might be implied in the development of this researcher profile, we should reflect on how researcher identity has been defined and empirically addressed and discuss the theoretical assumptions guiding researcher profile definition. Further, in undertaking this analysis, we recognized that identity has over time been under empirical and theoretical debate, particularly in organizational studies (Alvesson et al. 2008; Atewologun et al. 2017). We were attentive to these conversations in our study, acknowledging debates in specific domains, such as researcher identity, are influenced by and can modify discourses on identity in other domains.

\section{Framing the study}

In establishing a useful framework for the study, we started by looking at previous reviews on identity, both in general and specific domains other than the researcher identity domain and

\footnotetext{
${ }^{1}$ An international team of five researchers researching $\mathrm{PhD}$ and post-PhD identity development with various disciplinary backgrounds (psychology, education and sociology)
} 
higher education field-largely teacher, professional, mentoring or management identity (Epitropaki et al. 2017; Holck et al. 2016; Palmer et al. 2015; Trede et al. 2012; Van Lankveld et al. 2017). This first reading of the existing reviews revealed 'identity' in studies has often been ill-defined - not explicitly stated but implicit — and varied in its apparent focus. This fuzziness was largely evident only through a careful interpretive reading as noted by others (Alvesson et al. 2008; Atewologun et al. 2017; Brown 2017; Pifer and Baker 2013). This initial evidence was integrated with (a) our own experience as researchers in the field of early career researcher identity development (Castelló et al. 2015; McAlpine et al. 2013) and (b) a series of iterative readings of the articles included in this review. Two principles emerged as a result of combining these different sources of evidence which were central to how we approached the review: (a) research on identity can be framed across a range of meta-theories and (b) we can examine the manifestations of different meta-theories through different dimensions of identity. Both meta-theories and dimensions are defined as follows and elaborated in the 'Method' section.

Meta-theories As individuals, we each draw on different epistemological and ontological perspectives or ways of viewing the world, with our views providing a framework through which to understand human experience (Kuhn 1962). A meta-theory, an analytic lens ${ }^{2}$, provides 'a loose collection of logically related assumptions, concepts, or propositions that orient thinking and research' (Bogdan and Biklen 1998 p.22): more specifically, (a) a belief about the nature of knowledge, (b) a methodology and (c) criteria for validity (MacNaughton et al. 2001). Each meta-theory represents the philosophical intent or motivation for undertaking a study (Cohen et al. 2002).

Understood in this way, meta-theories act as knowledge-claims guiding decisions regarding research questions, conceptual frameworks and methodological decisions in empirical studies. Further, they are defined differently depending on disciplinary traditions. Thus, since research on identity has developed transversally from a varied range of disciplines, we did not expect or seek a shared, standard way to understand and classify the influence of different meta-theories on studies of identity (Atewologun et al. 2017). To capture this variation and organise the studies reviewed, the analytical structure that we developed integrated authors and traditions from sociology, psychology and education, the three main traditions that account for the variability of the studies included in this review (Bogdan and Biklen 1998; Kuhn 1962; Neuman 2000; Sousa 2010).

Our view is that meta-theories can be represented on a continuum. The continuum allows for a range of positions between two extremes as to what is considered valid knowledge. In other words, the continuum avoids suggesting discrete perspectives, but rather proposes overlaps and grey zones as to epistemological assumptions. In this continuum, we distinguished two distinctive end-points, and along the continuum variants that we refer to as metatheories, which depending on the disciplinary traditions might have different names. At one end of the continuum, we situated positivism - reality is directly observable and scientific knowledge is exclusively valid, with objectivity not only possible but also desirable in developing scientific work. Related meta-theories considered in different disciplinary traditions include cognitivism and behaviourism or associationism.

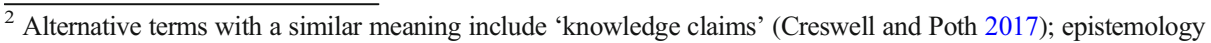
and ontology (Neuman 2000).
} 
The other end of the continuum is represented by post-positivism. Here, there is a great diversity of meta-theories but the major claim is that reality cannot be understood without taking into account the social context; thus, reality is subjective, and, to some extent, knowledge and scientific interpretations are situated within the experiences of the participants and researchers. Meta-theories move from more realistic and individual to more sociocultural and contextual premises and explanations of people, activity and concepts. Common metatheories include constructivism/interpretivism, transformative and critical realism.

Constructivism assumes reality is different depending on who interprets a specific situation or individual, which in turn, results in a spectrum of individually to more socially based explanations, theories and related notions. Agency and the notion of mental representation are usually related to the former while references to socio-historical and cultural mediation as well as to communities as tools for development are used by the later. Transformative aims to use research and derived interpretations to promote social change, given core notions of power relationships, justice or emancipation. A final emerging meta-theory is critical realism, which assumes many of the constructivist statements but claims ontological realism restricts the range of plausible interpretations.

These different meta-theories represent the underlying assumptions researchers use in planning, conducting and interpreting research, though they often remain implicit (Atewologun et al. 2017) and may or may not be used coherently. Moreover, meta-theories are constantly reframed and reinterpreted due to the implications of empirical findings, disciplinary cultures and constraints. However, meta-theories are not particular to the concept of identity; rather, they characterise a general way to understand research and related concepts. So, they were unlikely on their own to be productive in achieving our goal of differentiating stances towards identity. We needed a more fine-grained analytic tool.

Dimensions of identity We needed a concept-specific approach to evoke the underlying dimensions relevant to capturing the variability in how identity was used empirically. We chose to derive the specifics of these dimensions abductively since they needed to be distinctive enough to explain variability, an approach consistent with previous reviews from different disciplinary areas (Alvesson et al. 2008; Atewologun et al. 2017). Collectively, the application of these dimensions would provide a unique portrait of the characterization of identity for any one study — and potentially be related to the meta-theories - though we could not assume in what way. Our assumption was that the integrated assessment of the dimensions for each study would enable us to differentiate the studies' views as to the mechanisms claimed to explain the use of the concept of identity, relate these mechanisms with the meta-theories and, in turn, better understand the underlying assumptions of each study.

Based on results and considerations of previously mentioned reviews, theoretical frames of identity and the emergent characteristics of the articles included in our sample, we distinguished four emergent dimensions, with each understood as representing a continuum.

- The first dimension addresses the degree to which the view of identity varies in terms of its emphasis on the individual (Hermans 2001) through the social (Ivanič 1998; Zucchermaglio and Talamo 2000; Wenger 1999; Butler 1999).

- Dimension two highlights variation in the view of identity as stable through dynamic (Elliott 2005; Hermans 2001).

- Dimension three, in contrast, focuses on variation from a view of identity as singular (Ricoeur 1991) through to multiple identities held by one individual (Knez 2016). 
- The last dimension emphasizes the variation in focus from thinking (Archer 2000) through action in characterising identity (Vandenberghe 2007).

The four dimensions echo theoretical discussions around the notion of identity in varied disciplinary traditions in the last twenty years. In other words, the cultural, situational or psychological nature of identity has consistently encompassed monolithic through dualistic understandings of the self and boundaries of reality as physical through mental constructions (Hermans and Dimaggio 2007; Hammack 2015; Ibarra and Barbulescu 2010; Strauss 2017).

\section{Aim of review}

As noted earlier, we wanted to unpack the underpinnings of the notion of researcher identity, given its increasing, but unexamined, use in research (and policy). Such studies, if published, legitimate the conclusions and the uses of the research-based knowledge and thus, implicitly inform readers' understanding of researcher identity. This, in turn, has implications for future research, and for others using the research for policy or pedagogical purposes.

Our underlying assumption was that meta-theoretical assumptions (often implicit) guide both empirical research design and interpretation of findings (Bogdan and Biklan 1998) further, that we needed to incorporate dimensions of identity to generate a more fine-grained characterization of the varied stances to researcher identity. By making clear the underlying dimensions of identity in these studies, and assessing them as regards the relative weight given to the different meta-theories, we could identify variation and any gaps.

To unpack the theoretical underpinnings of researcher identity, we asked:

1. What is the range of ways in which 'researcher' identity is understood and used in empirical studies? Specifically,

a. What dimensions are used to define researcher identity?

b. How can we characterise the underlying meta-theories in relation to these dimensions?

2. What are the implications of this for our understanding of identity and future research?

\section{Method}

\section{Scope of the review}

The review included peer-reviewed journal articles in English, French and Spanish ${ }^{3}$ that were published from 1997 to 2017 and explored the identity of researchers at any stage of their career (e.g. doctoral students, senior researchers, etc.). The twenty-year window allowed for a comprehensive overview of the literature, while including papers in multiple languages extended the review to encompass multiple research cultures.

\footnotetext{
${ }^{3}$ We initially chose these languages because we did not want to restrict the search to only English published research, and these were the languages the authors could read. Neither French nor Spanish published papers remained in the final review.
} 
Inclusion criteria focused on articles addressing 'researcher identity,' but also other keywords such as 'disciplinary identity' or 'academic identity' as long as the main focus met our interest in researchers.

\section{Search terms and article filtering}

Web of Knowledge and SCOPUS were chosen as the two online databases underpinning our literature search. We began with the primary keyword, 'identity,' and its two synonyms, 'selfconcept' and 'self-perception,' and then identified additional 13 secondary keywords meant to capture our interest in researcher identity development: scholarly, academic, trajectory, development, journey, researcher, junior researcher, early career researcher*, $\mathrm{PhD}$, doctorate, doctoral, postdoc*, and masters. Results of the searches were input into Mendeley, and once duplicates were removed, yielded 554 articles (details of the search string can be found at the online resource 1_ESM_4.pdf)

Given our aim, to explore how researcher identity specifically is conceptualized in empirical studies, we excluded the following:

a) Those without a clear focus on researcher identity;

b) Those with a focus only on specific socio-demographic characteristics in particular predefined groups, such as race and gender, since, after reading them, since they did not address researcher identity;

c) Non-empirical articles such as reviews and position papers as the aim was to analyse how evidence was linked to the approaches adopted by empirical research;

d) Those that studied other identities, e.g. teacher; and

e) Those that were interventions, since the focus was on evaluating programs rather than understanding identity.

After reviewing the abstracts, a significant number of articles were excluded: 342 were unrelated to researcher identity (e.g. papers from history or medical fields, studies on teacher education, etc.) and 98 focused on identities of specific groups, not on researcher identity (e.g. Chinese socialisation in US universities, scientific visibility of Mexican researchers, gender and academic socialisation). The remaining 114 papers were downloaded, and the complete text for each was read to assess its alignment with the inclusion and exclusion criteria and the objectives of this review. After careful examination, 41 articles were rejected because, although they mentioned researcher identity in the abstract, and upon reading the papers, it became clear that this was not the focus of the study (e.g. research paradigms, researcher health, otherness, researcher interactions, etc.), and 10 more were excluded because they were theoretical or position papers.

As well, 14 studies exploring writer, teacher or student identity, but not researcher identity were excluded. Finally, those articles assessing interventions aimed at promoting researcher identity were also excluded $(n=11)$. After applying these criteria, 38 articles were in the final selection and analysed in more depth (see Fig. 1).

\section{Teamwork}

An interesting part of co-authoring this literature review was that, although we shared a keen interest in researcher identity, our stance as to its various dimensions varied. As suggested by 


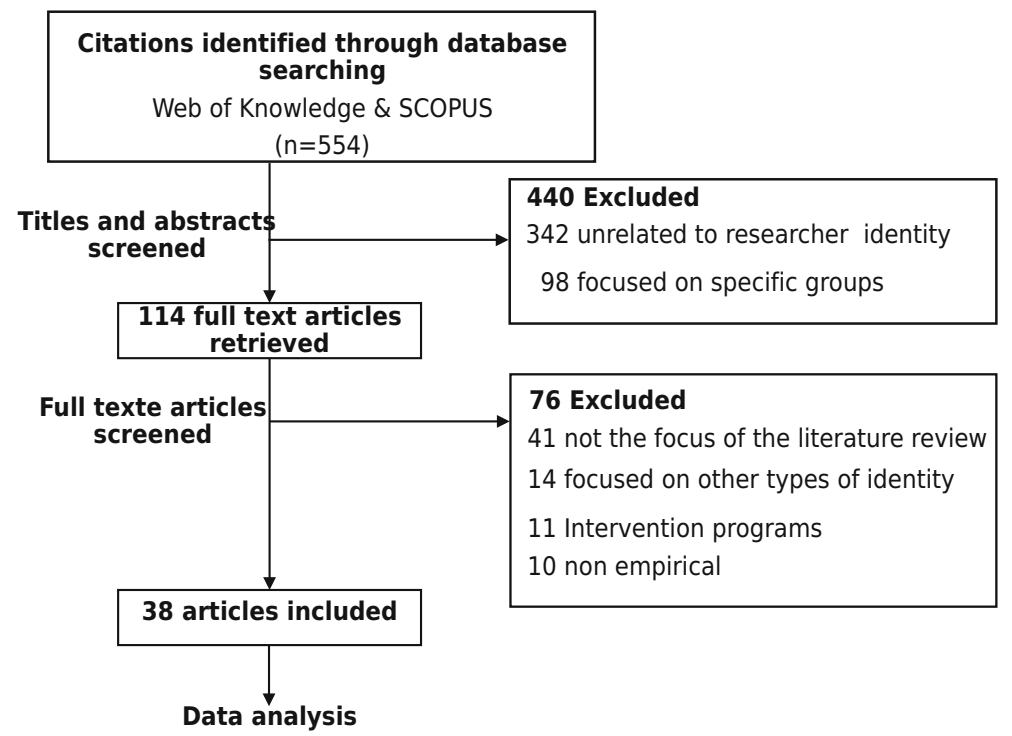

Fig. 1 Flow chart of the article filtering process

Gough et al. (2017), keeping this in mind eventually helped us to develop and fine-tune comprehensive definitions of researcher identity dimensions. We adopted an iterative procedure in which each of us read, coded and classified the selected set of articles individually. We then compared our results and reconciled them. The same process was subsequently conducted in pairs. To ensure that we shared the same definitions, and maintained consistency, we followed every round of analysis with thorough discussions about how the dimensions of researcher identity were represented in each article. Specifically, we reviewed any disagreements or different opinions and modified our definitions accordingly.

\section{Analysis}

The analysis took place in four steps, conducted by all the researchers as described in Teamwork.

First, the final selected sample of articles was read iteratively, and the following characteristics were documented in an excel spreadsheet: Author(s), year, definition of identity, keywords, aims of the study, design and results. For each study, relevant concepts related to conceptual approach, methodological approach, and significance were identified and entered in the spreadsheet. This first phase was non-inferential since the words and concepts identified were the same used in the articles.

Second, to assign an article to a particular meta-theory, we relied on the initial analysis of concepts and keywords involved in the analysed articles, thus keeping a low inferential level. Discussions focused on how these concepts and keywords were related to the four broad metatheories defined previously: each sustaining different purposes and notions involved (see Table 1 for a description of this analytical tool). In other words, once we agreed regarding the relationships between key notions and meta-theories, we applied the first analytical tool situating the articles along the continuum of meta-theories. Each researcher individually coded 
Table 1 Analytical tool to analyse the meta-theories underlying the studies

\begin{tabular}{|c|c|c|}
\hline \multicolumn{2}{|c|}{ Continuum of meta-theories } & $\begin{array}{l}\text { Concepts and Keywords involved } \\
\text { (examples from articles analysed) }\end{array}$ \\
\hline \multirow{11}{*}{ Positivism } & Cognitive & Objectivity \\
\hline & Associationism & Deductive logic \\
\hline & & Experiment \\
\hline & Critical realism & Ontological realism \\
\hline & & Epistemic relativism \\
\hline & & Judgemental rationality \\
\hline & Interpretivist/ & Situated cognition and learning \\
\hline & Constructivist & Socio-cultural differences \\
\hline & & Socially-constructed meanings \\
\hline & & Communities of practice \\
\hline & & Relativism \\
\hline \multirow[t]{3}{*}{ Post-positivism } & Transformative & Power \\
\hline & & Inequality \\
\hline & & Social Justice \\
\hline
\end{tabular}

each of the 38 articles. To guarantee reliability among coders, a series of paired analysis using Kappa's Cohen index were calculated. Results indicated agreement among researchers ranged from high to acceptable (0.79-0.88). The meta-theories provided an overview regarding how the study of identity as a whole was addressed.

Third, we developed a second analytical tool to provide a more nuanced picture of the results. Since the previous tool focused on the underlying assumptions of the study, not the specifics of how 'identity' was conceived, this tool differentiated variability as to how identity was either elaborated in the studies or represented in the description of the findings. It identified (a) those distinctive dimensions in defining identity, and (b) the continuum of characteristics or values differentiated in each dimension.

Dimensions and their values were defined and redefined until they covered all the variation regarding the range of interpretations of identity we found in the empirical studies reviewed (Table 2). Five successive rounds of analysis were performed in which all the researchers independently analysed a small group of articles $(6,6,9,9,12)$ and individual results were discussed until consensus was reached, either to introduce some modifications into the tool or to classify the article. Once consensus was reached regarding the meaning of each of the dimensions and their values, all the papers were analysed again independently by the researchers - with reliability assessed. To do this analysis, we first looked for key notions mainly in the introduction section. When not explicit, other related aspects, explanatory statements in the rest of the article, including results and discussion, were also reviewed. Finally, the whole article was checked to guarantee the validity of categories. The level of agreement ranged between 0.81 and 0.92 depending on the dimension and the coders, which is considered high for this type of analysis (Cohen et al. 2002).

Finally, we looked for relationships and correlations (a) among the dimensions and (b) the dimensions and the meta-theories. This final analysis enabled us to assess (a) differences in how these articles characterised identity along the dimensions, and (b) to 


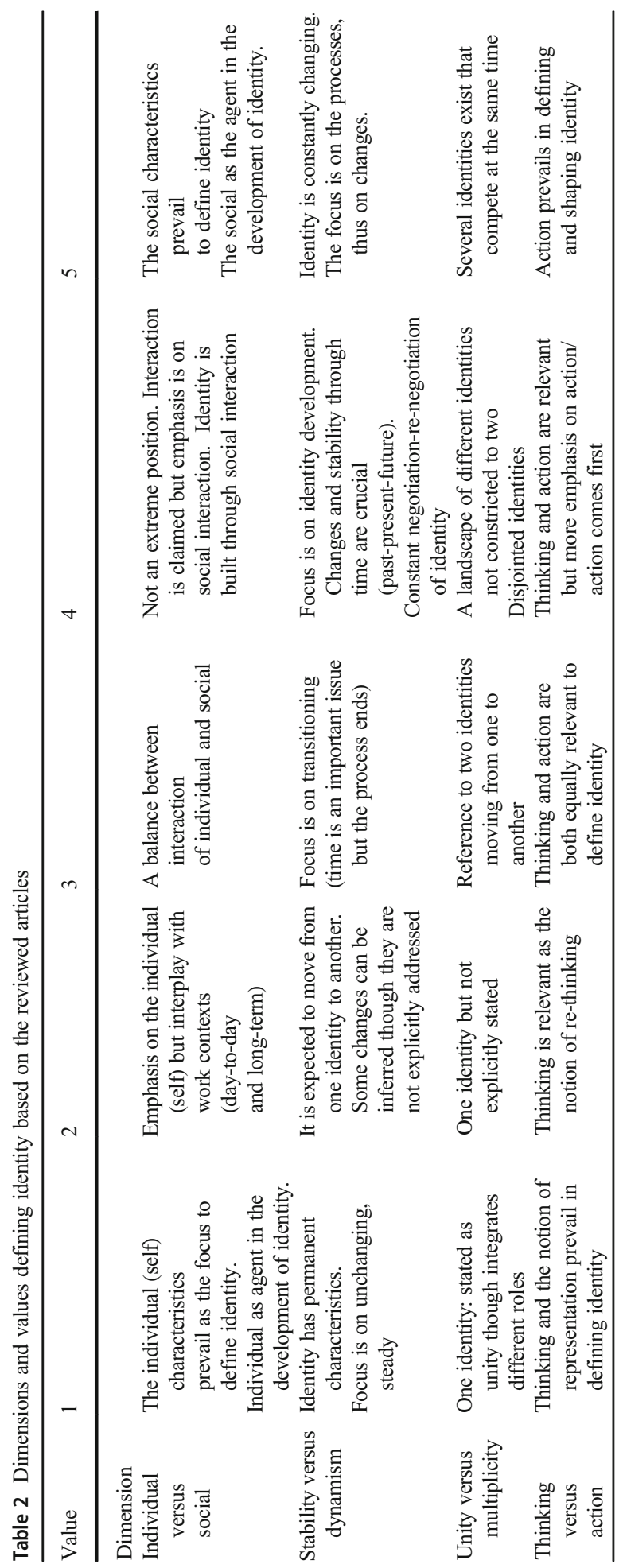


what extent there was a relationship between the understanding of identity, and (c) the meta-theory framing the study The final step in this analysis was to characterise qualitative clusters based on the identified regularities and consistent patterns. Clusters were primarily based on the correlations among the dimensions whereas secondary analysis looked for their relationship with meta-theories.

\section{Results}

\section{Descriptive characteristics of the studies}

Although our literature search covered the last 20 years, those articles fulfilling the inclusion criteria ranged from 2004 to 2017 and numbers tended to increase with time, especially from 2012 (see Annex 1). Regarding the journals, the scattering of the results is revealing $23 \%(n=6)$ of the studies were published in Studies of Higher Education; $15 \%(n=4)$ in Studies in Continuing Education; $11.5 \%(n=3)$ in Innovations in Education and Teaching International and 7.7\% $(n=2)$ in the International Journal of Doctoral Studies and Teaching in Higher Education. The rest of the journals $(n=9$, $35.1 \%$ ) have one single article published on the topic of researcher identity in the analysed period. Slightly more than half of the studies (58\%) were exclusively devoted to the development of researcher identity in doctoral students, with the remainder focusing on early career researchers $(28 \%)$ and experienced researchers $(14 \%)$.

\section{Meta-theoretical frameworks of studies}

Studies were distributed among three of the four considered meta-theories. Three quarters ( $76 \%, n=29)$ of the articles were classified as interpretative/constructivist. Of the remaining studies, $18.5 \%(n=7)$ were classified as critical realism and only $5.5 \%(n=2)$ as transformative. There were no examples of positivism (e.g. cognitivism).

Constructivist studies tended to situate themselves within a variety of theories noted earlier, which range from what can be broadly termed the sociocultural perspective of learning and development to other more sociological-related approaches. In all cases, historical and contextrelated issues are crucial to defining identity, typically understood as dynamic and a developmental process.

Studies representative of the critical realism framework were less diverse and relied on the idea of identity as a dynamic biographical process grounded in a history, with pre-existing personal understandings, which in turn influences the present interpretations and future learning (McAlpine et al. 2013). The notions of agency and trajectory are central to understand identity within this framework, which provide a means to balance unity and change.

The transformative framework is characterised by studies in which, in these cases, a feminist approach is used to characterise identity as dynamic, multidimensional, complex and socially developed (Rockinson-Szapkiw et al. 2017).

\section{Dimensions to define identity}

Within the four dimensions and their emergent values, we found a diverse distribution explaining the variation of all the articles included in this review (see Table 3). 
Table 3 Results regarding dimensions and values defining identity in the reviewed articles

\begin{tabular}{|c|c|c|c|c|c|c|c|}
\hline Value & 1 & 2 & 3 & 4 & 5 & NA* & Value \\
\hline Dimension & & & & & & & Dimension \\
\hline Individual & - & $19 \%$ & $10 \%$ & $61 \%$ & $10 \%$ & & Social \\
\hline Stability & - & $3 \%$ & $24 \%$ & $55 \%$ & $18 \%$ & & Dynamism \\
\hline Unity & $16 \%$ & $8 \%$ & $34 \%$ & $19 \%$ & $23 \%$ & & Multiplicity \\
\hline Thinking & $5 \%$ & $5 \%$ & $16 \%$ & $51 \%$ & $13 \%$ & $10 \%$ & Action \\
\hline
\end{tabular}

*NA, non-applicable

\section{Individual versus social}

Most of the studies assumed that identity is socially constructed $(71 \%, n=27)$ (located to the right end of the continuum, positions 4 and 5) and though individual's experiences are acknowledged, the role that authors attribute to them is diverse. In some cases, identity is considered relational and individuals are claimed to position - and be positioned by othersdifferently in particular changing scenarios (Castelló et al. 2013; Cotterall 2015; MurakamiRamalho et al. 2013). In other cases, the emphasis is placed entirely on the role of sociocultural, historical and political contexts in which situational identities are negotiated and lived in and through activity (Gunasekara 2007; Remich et al. 2016).

Studies that assumed identity is mainly individually based, thus located close to the left end of the grid continuum, were less frequent $(19 \%, n=7$, position 2$)$. They emphasised the role of individuals, and especially their agency in the development of identity, without denying the importance of context and the situated nature of this development (Gardner and Willey 2018; Inouye and McAlpine 2017; McAlpine et al. 2014). These studies (position 2) mostly represented critical realism, except two (Buss et al. 2014; Pifer and Baker 2016), which, despite adopting a constructivist perspective, considered the construction of the self, through notions as 'salience' or 'learned mind' and 'perceptions of the self' respectively, as a matter of individuals rather than a social issue.

Finally, only 4 studies were situated in the middle of the continuum (3), meaning that identity resulted from balancing individual agency and social influence. These studies defined identity by using expressions such 'a set representation built by individuals in sociocultural contexts' (González et al. 2014) or 'the core sense of the self' (Rockinson-Szapkiw et al. 2017).

\section{Stability versus dynamism}

Most of the studies $(73 \%, n=28)$ considered dynamism a crucial dimension of researcher identity, rather than understanding identity as a stable characteristic of individuals (positions 4 and 5 of the continuum). Most of them $(n=21)$ focused on identity development, and thus, looked for changes through time. In some cases, development is understood explicitly as constant negotiation-re-negotiation of past, present and future identity experiences, within the notion of identity-trajectory (McAlpine et al. 2014; Inouye and McAlpine 2017); development is a relevant heuristic to characterise dynamism in the continuous negotiation between stability and change through time. The other seven studies, which consider researcher identity as a dynamic construct, were situated at the end of the continuum and thus made more explicit 
claims about identity being constantly changing, or even fluid (Gunasekara 2007; Rayner et al. 2015). This stance requires authors to focus on processes instead on outputs.

Almost a third $(27 \%, n=10)$ of the studies took a balanced position on this dimension namely they focused on transitioning from one identity to another (Dison 2004; González et al. 2014; Holley 2015) or modifying a particular identity. In these cases, the process of change is expected to end, whereas this is not the case for the rest.

\section{Unity versus multiplicity}

Around a quarter of the studies $(24 \%, n=9)$ explicitly stated individuals have one single identity, though it might integrate several roles (positions 1 and 2). When combined with the notion of identity-trajectory, unity is the result of the continuous negotiation between stability and change that characterised the former dimension (Gardner and Willey 2018; Inouye and McAlpine 2017; McAlpine et al. 2014; McAlpine and Amundsen 2009).

More than one third of the studies $(34 \%, n=13)$ referred to two identities and focused on moving from one to another. Transitioning from a student to a researcher identity is one common topic within this set of studies, as well as from teacher to researcher identity (Baker and Pifer 2011). Consequently, although time is important, what is expected and looked for is the end of the process of identity change (González et al. 2014; Holley 2015). However, several studies included in this group did not provide an explicit definition of identity except for describing the transition situation in which students (or professionals) have to develop their identity as researchers (Araújo 2009; Baker and Pifer 2011; Heinrich 2005; MurakamiRamalho et al. 2013; Rayner et al. 2015).

Finally, the largest set of studies $(42 \%, n=16)$ accepted, more or less explicitly, a landscape of different identities not necessarily restricted to two (positions 4 and 5). Within this set, almost half of the studies $(n=7)$ focused on contradictions and conflicts that arise when individuals negotiate particular identities related to their participation in different contexts. In such cases, their stances and activities are not compatible with the research identity they are developing (Castelló et al. 2013; Cotterall 2015; Mewburn 2011). The focus is on relationships between these identities, or identity dimensions (Rockinson-Szapkiw et al. 2017), roles (Merolla and Serpe 2013; Pifer and Baker 2016) or identity positions (Castelló et al. 2013). The aim is to elucidate why, when and how conflicts are solved; norms and practices are adopted, ignored or resisted (Hökkä, Eteläpelto and Rasku-Puttonen 2012); and how multiple trajectories are linked to varying membership positions in multiple communities (Smith and Boyd 2012; Zambo et al. 2015). The rest of the studies $(n=8)$ were more situated at position 5 of the continuum and assume identities are not only multiple because they are situational (Guerin 2013; Gunasekara 2007) but they are also disjointed (Costa, 2015).

\section{Thinking versus action}

Studies located in positions 1 and 2 of this continuum assumed that thinking, thus ideas, representations, conceptions or perceptions, prevails in defining identity. This means that, although practices and experiences are acknowledged as highly relevant, the way in which individuals perceive and interpret these experiences is also crucial to explain how identity is developed and shaped. These few studies $(n=4,6 \%)$ emphasised the role of self-reflection and critical thinking (Alexander et al. 2014; Leibowitz et al. 2014) as well 
as the thoughts, ideas or representations of oneself, which are constructed in social contexts (Buss et al. 2014; González et al. 2014).

A slightly higher number of studies $(n=6,15 \%)$ took an intermediate stance regarding the role of thinking and action in constituting identity. They assumed some inner processes filter or mediate the activity and contextual factors that, in turn, influence the self-perceptions of identities and the relationships between each. These instances ranged from meaning-making mechanisms (Rockinson-Szapkiw et al. 2017), self-definition and personal history (McGregor et al. 2010; Schulze 2014), writing and self-narratives (Cotterall 2015) to individuals' internal expectations useful to make sense of themselves (Hökkä et al. 2012).

Finally, the majority of studies were situated in the action end of the continuum (positions 4 and 5), thus assuming that thinking and action are relevant but placed more emphasis on action $(50 \%, n=19)$, or that action prevails in defining and shaping identity $(13 \%, n=5)$. In these studies, identity is shaped or enacted by participation in different contexts (Castelló et al. 2013; Gardner and Willey 2016; Inouye and McAlpine 2017; McAlpine et al. 2013, 2014; McAlpine and Lucas 2011; Merolla and Serpe 2013; Wegener et al. 2016). Another view is that activity or behaviour comes first, and researcher identity is developed by - and visible in - how one speaks, reads, writes or behaves (Baker and Pifer 2011; Guerin 2013; Thompson et al. 2016). Some of these studies adopted the notion of participatory social practices (Lave and Wenger 1991; Wenger 1999) in which identity is shaped by Communities of Practice (Boyd and Smith 2016; Dison 2004; Lassig et al. 2013). There were four articles (10\%) (Araújo 2009; Costa 2015; Gunasekara 2007; Pifer and Baker 2016) that could not be classified in regards to this dimension since they did not offer any explanation regarding this dimension.

\section{Relationship among dimensions and meta-theoretical frameworks in the researcher identity studies}

In looking at relationships among the dimensions and between the dimensions and the metatheoretical approaches, one finding predominated. Two dimensions moved consistently together: the second, stability vs dynamism, and the third, unity vs multiplicity. After collapsing the data from the rubric into three values, (1) low (levels 1 and 2 of the former classification), (2) medium (former level 3) and (3) high (former levels 4 and 5), this consistency was remarkable (see Table 4). Four different clusters were identified based on the primary commonalities in the dimensions of stability vs dynamism and unity vs multiplicity and related variation on the rest of the dimensions defining identity. We provide both qualitative and quantitative characterizations for each cluster.

The dimensions are key to understand the distinctiveness of each cluster, but they alone do not provide information about the prevalence of the different clusters or the prevailing stance within each cluster - essential if one is to understand how the construct is being empirically used in the field, which was obtained by looking at the meta-theoretical approaches distribution across clusters.

\section{Cluster 1. Transitioning among identities}

The fourteen papers included in this cluster are characterised by understanding identity as socially constructed and dynamic and accept, though not always explicitly, the existence of more than one identity, even multiple identities, which in some cases, can be disjointed. The notion of transitioning among different, often competing, identities is discussed as both a 


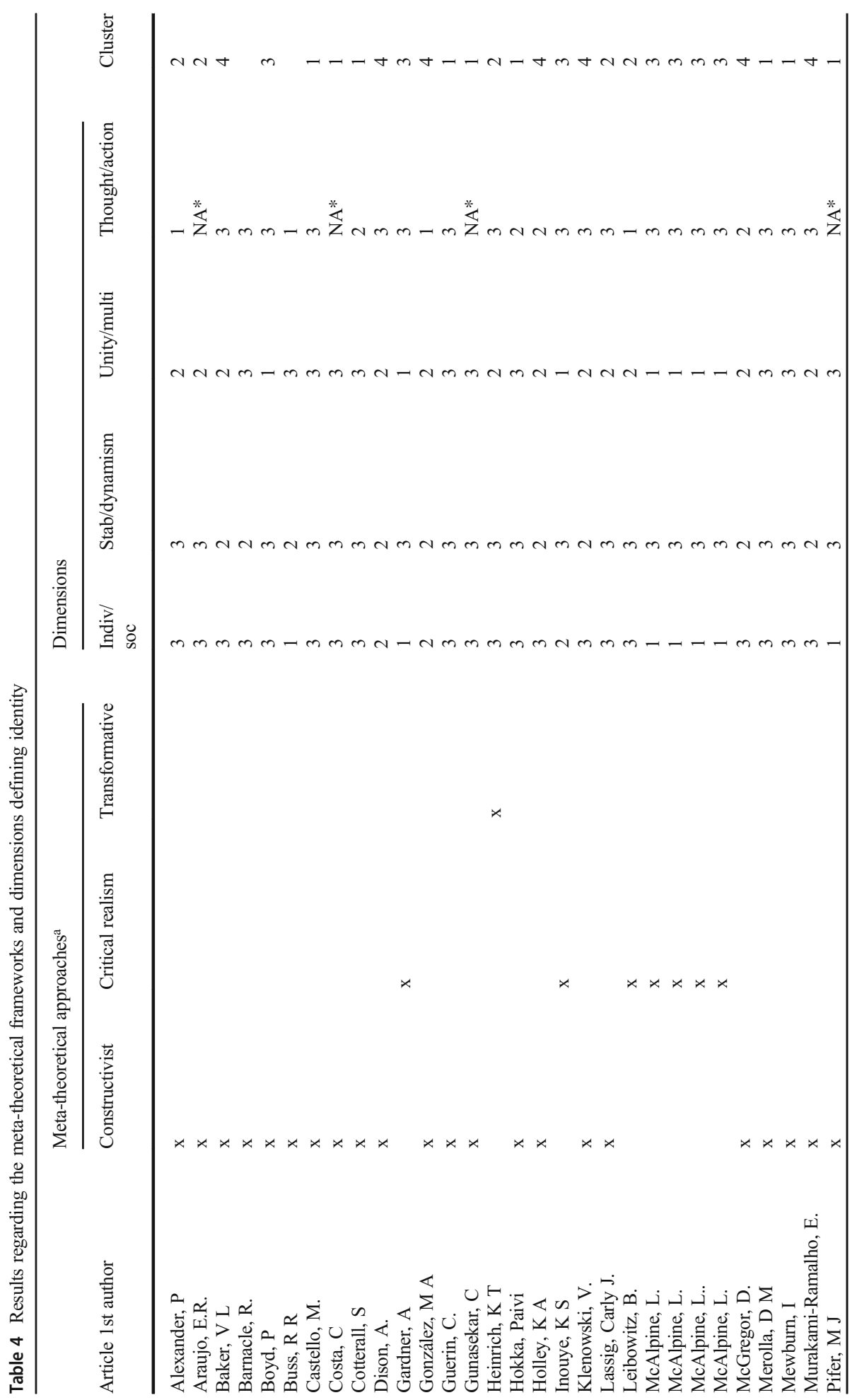




$$
\sqrt{1}
$$


theoretical assumption and a focus for the empirical data collection and analysis. Moreover, identity is mainly socially constructed and developed, except for Pifer and Baker (2016) who emphasised individual characteristics as the focus to define identity, and Rockinson-Szapkiw et al. (2017) who were located in the middle, thus valuing both the individual (self) and contextual influences in identity development. More than half of the papers included in this cluster considered both thinking and action as relevant but placed more emphasis on action in defining and shaping identity. Of the remaining, only four took an intermediate stance, thus claiming that both thinking and action are relevant to define identity. The remaining three did not reveal a clear stance on this dimension.

Interestingly, all papers in this cluster were constructivist in approach. The articles in this cluster were also quite recent, all published in the last seven years (the first one is from 2011).

\section{Cluster 2. Balancing identity continuity and change}

Six papers that, as in the first cluster, considered identity as socially constructed and highly dynamic shape the second cluster. The main difference from the previous group relates to their stance towards unity in identity definition. Most of the papers in this cluster only referred to one single identity (Araújo 2009) or to moving from one identity to another (Lassig et al. 2013). In this latter case, the process is sequential, and no multiple identities are considered simultaneously except for some specific transition moments (Alexander et al. 2014). However, transitions were rarely the explicit focus of the studies included in this group. Despite the consistency of identity as socially constructed and dynamic, there is great variability regarding whether thought or action prevails in defining identity. Half the papers felt action and participatory practices prevail in shaping and defining identity, whereas, within the other half, two mentioned representations and individual thoughts in identity definition and the last provided no information regarding this dimension (see Table 4).

All the papers included in this cluster were in the post-positivist meta-theoretical approaches. However, they were theoretically highly variable with all three meta-theoretical approaches represented. Publication year ranged from 2005 to 2015.

\section{Cluster 3. Personal identity development through time}

The eight papers gathered in this cluster were highly consistent in defining identity as dynamic and mainly individually driven, thus a developmental and agentive process - while acknowledging the interaction with not only social contexts but also physical contexts (McAlpine and Lucas 2011). Only in two cases (Boyd and Smith 2016; Wegener et al. 2016) did social characteristics prevail. All of them also defined identity as unique and driven by action and participation on social events.

As regards their meta-theoretical stance, the majority shared critical realism, with only the two favouring social characteristics located in the constructivist. Years of publication range from 2009 to 2017.

\section{Cluster 4. Personal and stable identity}

The last cluster gathers seven papers defining identity as mainly unique and stable, though some changes can be inferred in transitions. They viewed social characteristics as crucial or important in defining identity. Still, there was greater variability observed regarding the role of 
action. Four considered identity as mainly shaped through action and participatory practices; two claimed that thinking and action are both equally relevant to define identity (Klenowski et al. 2011; Murakami-Ramalho et al. 2013) and the last (González et al. 2014) situated thinking and the notion of conceptions, strategies and feelings as predominant.

All the studies in this cluster shared the constructivist framework and were published between 2010 and 2015 .

Finally, we were unable to locate three studies in any cluster since they did not display any regularities related to the dimensions we applied in this review. Two of them display a balanced position regarding the dimension of stability and change but claim for multiple identities: Barnacle and Mewburn (2010) argue the social nature of identity and the relevance of action; Buss et al. (2014) that identity is individually based and guided by personal thoughts and mental representations. The last defines identity as unique and stable but shaped through social and action (Thompson et al. 2016).

\section{Emerging patterns across clusters}

\section{Perspectives on dynamism}

Using the dimensions to define identity revealed that most papers looked at dynamism and movement of identities, consistent with the constant changes researchers face nowadays. This dynamism related to three different characteristics: transitions, development and fluidity.

In the papers interested in transitions, dynamism is restricted to changes among identities or roles. Thus, it is expected to end, usually when the new identity replaces a former one. Contradictions may be considered, especially when the focus is transitions, with a single changed identity conceived as ensuring self-coherence and stability. In contrast, in those papers focusing on identity development, no ending or replacement of one identity by another is expected. In these cases, time plays a crucial role to explain how and why specific identity characteristics are characterised. Here, dynamism constitutes constant change, with identity understood as fluid, and may include the notion of multiple identities, even disjointed. Regardless, in both cases, longitudinal designs and trajectories are valued.

\section{Perspectives on action}

Papers also displayed alternative understandings of the role of action in shaping identity: whether related to activity or the community. Those papers that related action to activity considered the development of identity linked to the individual's appropriation of the specific characteristics of a particular type of activity (writing, talking, behaving). In contrast, other papers assumed that the characteristics of a particular community and its practices shape identity. This difference is not minor since results show a relationship between the notion of action and the nature of identity as social or individual. Papers exploring particular contexts of researcher identity development (e.g. engineering researchers) were those that more frequently attributed the individual a substantial role in defining its identity (or at least equated the role of the individual with the social). In contrast, those studies assuming that communities of practice shape identity defined identity mainly as socially constructed. 


\section{Interaction of dimensions and meta-theories}

The four clusters identified illustrate interesting relationships among and between the dimensions and the meta-theoretical approaches displayed by the reviewed studies. Looking at the results, the consideration of identity as unique or multiple is the main difference between the two first clusters (transitioning among identities and balancing continuity and change); thus, papers included in both agree that researcher identity is socially constructed and dynamic. However, papers in the first cluster, claiming multiple identities, were the most frequent and recent ones, and were mainly situated in socio-cultural constructivist approaches.

Differences in the remaining clusters related to both dynamism and the social or individual nature of identity. Papers in the third cluster (personal identity development through time) claim that changes and dynamism are linked to the notion of identity development; at the same time, they situate the self and agency as key terms to explain changes through time. Theoretical consistency is high in this group, which leads us to conclude that when it comes to researcher identity, the critical realism meta-theory assumes identity as a personal developmental process, mainly individual.

In contrast, those in the fourth cluster (personal and stable identity) considered identity as mainly stable and personal and, though the influence of social characteristics in its development is not denied, the role attributed to the social and its theoretical understanding may vary significantly in this group. Theoretically, this group represents the more individually based explanations within the constructivist meta-theory, assuming agency and situated mental representations as crucial in researcher identity.

\section{Discussion}

We conducted this review to unpack the underpinnings of 'researcher identity,' a notion used and debated in different disciplinary fields (e.g. educational and social psychology, organizational studies), particularly in higher education and faculty or academic identity development (Alvesson et al. 2008; Atewologun et al. 2017; Brown 2017). While we had presumed that studies focusing on a specific notion-such as researcher identity-would clarify their epistemological and theoretical assumptions, this proved not to be the case.

Thus, we used an analysis that integrated meta-theories of scientific knowledge with dimensions of identity to interpret the studies. We identified four stances towards researcher identity. These stances incorporate intriguing nuances and complex characterizations; particularly highlighting difference perspectives on (a) dynamism, (b) action and (c) the interaction of dimensions and meta-theories - with implications for both our understanding of researcher identity and future research.

Important nuances emerged when analysing how each paper explained those characteristics and dimensions defining researcher identity. The results as a whole revealed the prevalence of meta-theories towards the post-positivist end of the continuum with the premises linked to constructivist meta-theoretical approaches prevailing across the papers. Further, all four stances incorporated papers with a constructivist approach. Thus, while the meta-theory was necessary to understand the approach to the study, it was insufficient to characterise the researcher identity stance. Notably, none of the analysed papers adopted a positivist stance - presumably because a positivist stance does not engage with the subjectivity of the researcher, and thus, the notion of identity has no value or interest per se. 
As regards the four characterizations of researcher identity, their value lies in two directions. The first is to enable fine-grained comparisons of already-published empirical studies: to judge and contest the ways in which different stances may provide different perspectives (and blind-spots) on researcher identity. The second is the valuable tool that the four dimensions represent for future research. Researchers can use them to characterise and report their own stance. Further, if this were done consistently, over time, we would generate a collective empirical understanding of researcher identity that would be much more nuanced, complex and comprehensive.

Three further considerations emerge that have implications for our current understanding and future research on researcher identity. First, given the limited number of authors that appear more than once in the final articles, we wonder if some authors delve into identity as an explanation of other areas of interest rather than as an intrinsic interest, i.e. identity as peripheral rather than central. In other words, what exactly is being looked at when the notion of researcher identity is used? Related subjects, such as $\mathrm{PhD}$ programs, attrition, professional development or career changes, were at the core of some of the reviewed studies. In these cases, identity was used as a way to interpret the results or even as a heuristic to conceptually frame those research subjects. The theoretical approaches assumed by those papers appeared to come from disciplinary research fields not always consistent with the researcher identity definition they claimed. This might explain why authors used some theoretically grounded notions that were in opposition (e.g. 'identities' in plural alongside 'transition'), or researchers might avoid the term identity totally given the difficulty of clarifying it in an empirical paper. A final explanation for this lack of clarity might be theoretical shifts in researcher identity that cannot be detailed in an empirical paper.

Second, we suggest current and future researchers of identity can use the dimensions to clarify their underlying assumptions and the implications for their empirical designs and methods. For instance, reflecting on our understanding of stability and change helps decide how to address development in defining identity. Further, clarifying to what extent identity is individual or socially constructed forces us to consider notions of structure and agency. Focusing on the thought-action dimension implies taking a stance on how and when change occurs as well as how the concept of action is related to individual and communities. Finally, reflection on whether identity is unique or multiple forces us to relate researcher identity to roles and spheres of activity. We argue that using such an approach would legitimate the conclusions and uses of the research-based knowledge and better inform readers' understanding of researcher identity. This particularly has implications for others using the research such as developers, curriculum designers and policy makers.

Third, others, regardless of social science discipline, could use the same systematic analytic procedure - dimensions and meta-theoretical approaches - to characterise the representation of more abstract concepts in empirical studies.

\section{Limitations}

Some decisions we made entailed limitations. Restricting the review to the notion of researcher identity could have biased some results, especially as regards the notion of the multiple identities since this decision may have excluded some pertinent papers. However, we did not consider using the broader search term 'identity' without any secondary keyword, because this would have broadened the scope and focus of the review too much. 
We also excluded articles focused on the identity of specific groups but not on researcher identity. While this could have resulted in missing articles, based on the initial reading, this was not the case for any of the discarded articles. Still, the final sample included four papers addressing early career researcher identity as intersecting with other factors like gender. Though we acknowledge discussing intersectionality might have resulted in more nuanced researcher identity explanations in those four cases, examining this issue in sufficient depth would reframe the focus of the paper.

Readers might also wonder whether some form of selection bias contributed to the prominence of social constructivism with less than a quarter representing other meta-theories. We believe this finding stems from the predominant use of social constructivism in the general literature on early career researchers.

Another aspect of selection bias that the final articles reviewed were all English may reflect the databases used. Van Leeuwen (2013) argues that WoS and Scopus do not consistently incorporate the literature in the social sciences and humanities and have a bias towards English studies. Further, powerful cultural/linguistic differences may lead to different ways of understanding early career researcher experience. These limitations can be explored in future reviews.

Finally, a further limitation stemmed from our decision to not discuss the relationships between methodologies, theoretical stances and framings of identity, as those elements are often intimately interrelated. Such a discussion might have enhanced the contribution of this study, but we felt it was beyond the scope of the review's focus on the framing of identity.

\section{Future research}

Many questions still remain: To what extent is the interpretation of data influenced by the researcher identity stance, whether or not explicit? What are we not seeing when interpreting data through our own stance only? Are we clear on why we are adopting one stance and not another and for what goal? What influence might data collection and analysis be having on our understanding of identity; recall that those incorporating dynamism often used longitudinal designs. Perhaps the biggest: To what extent and under what conditions is identity a productive notion for understanding early career researcher experience? For researchers in this field (like us), these questions provoke considerable thought since if we do not answer them, our use of the notion of research identity may not prove as productive as it might.

\section{Conclusion}

We began this paper noting that identity is a frequently used and contested concept. The results from the review make clear why this may be the case, given the range of different perspectives on identity in the studies analysed. Interestingly, we noted few attempts to contrast the stance taken with other studies, yet such comparison is important if we are truly to make sense of empirical findings.

Further, the unique results from this review clarify how particular understandings of researcher identity relate to a set of underlying conceptual dimensions and theoretical stances. Through the analytical tools used, we drew out the nuances and complex conceptualisations when studies address researcher identity empirically. Given that many studies had not clearly expressed a stance towards identity, a major contribution of our analysis was to clarify not only 
the specific ways in which identity was understood in each study, but also to characterise how identity was generally conceived in the researcher identity literature. In the process, we created a discourse for articulating stances towards identity. We hope the analysis and the discourse lead to fruitful debates among researcher identity scholars.

Funding Information European Commission. Directorate-General for Education and Culture Erasmus+ (2017-1-ES01-KA203-038303)

European Cooperation in Science and Technology (COST Action IS1401)

Open Access This article is licensed under a Creative Commons Attribution 4.0 International License, which permits use, sharing, adaptation, distribution and reproduction in any medium or format, as long as you give appropriate credit to the original author(s) and the source, provide a link to the Creative Commons licence, and indicate if changes were made. The images or other third party material in this article are included in the article's Creative Commons licence, unless indicated otherwise in a credit line to the material. If material is not included in the article's Creative Commons licence and your intended use is not permitted by statutory regulation or exceeds the permitted use, you will need to obtain permission directly from the copyright holder. To view a copy of this licence, visit http://creativecommons.org/licenses/by/4.0/.

\section{References}

Alexander, P., Harris-Huemmert, S., \& McAlpine, L. (2014). Tools for reflection on the academic identities of doctoral students. International Journal for Academic Development, 19(3), 162-173.

Alvesson, M., Ashcraft, K., \& Thomas, R. (2008). Identity matters: reflections on the construction of identity scholarship in organization studies. Organization, 15(1), 5-28.

*Araújo, E. R. (2009). 'With a rope around their neck': grant researchers living in suspended time. New Technology, Work and Employment, 24(3), 230-242.

Archer, M. S. (2000). Being human: the problem of agency. Cambridge University Press.

Atewologun, D., Kutzer, R., Doldor, E., Anderson, D., \& Sealy, R. (2017). Individual-level foci of identification at work: a systematic review of the literature. International Journal of Management Reviews, 19(3), 273295.

Baker, V. L., \& Lattuca, L. R. (2010). Developmental networks and learning: toward an interdisciplinary perspective on identity development during doctoral study. Studies in Higher Education, 35(7), 807-827.

*Baker, V. L., \& Pifer, M. J. (2011). The role of relationships in the transition from doctoral student to independent scholar. Studies in Continuing Education, 33(1), 5-17.

*Barnacle, R., \& Mewburn, I. (2010). Learning networks and the journey of 'becoming doctor'. Studies in Higher education, 35(4), 433-444.

Bogdan, R. C., \& Biklen, S. K. (1998). Foundations of qualitative research in education. Qualitative research in education: an introduction to theory and methods, 1-48.

*Boyd, P., \& Smith, C. (2016). The contemporary academic: orientation towards research work and researcher identity of higher education lecturers in the health professions. Studies in Higher Education, 41(4), 678-695.

Brown, A. D. (2017). Identity work and organizational identification. International Journal of Management Reviews, 19(3), 296-317.

*Buss, R., Zambo, R., Zambo, D., \& Williams, T. (2014). Developing researching professionals in an EdD program: from learners and leaders to scholarly and influential practitioners. Higher Education, Skills and Work-based Learning, 4(2), 137-160.

Butler, J. (1999). Gender trouble: feminism and the subversion of identity. New York: Routledge.

Castelló, M., Iñesta, A., \& Corcelles, M. (2013). Learning to write a research article: ph. D. Students' transitions toward disciplinary writing regulation. Research in the Teaching of English, 442-477.

Castelló, M., Kobayashi, S., McGinn, M. K., Pechar, H., Vekkaila, J., \& Wisker, G. (2015). Researcher identity in transition: signals to identify and manage spheres of activity in a risk-career. Frontline Learning Research, 3(3), 39-54.

Cohen, L., Manion, L., \& Morrison, K. (2002). Research methods in education. Routledge.

*Costa, C. (2015). Outcasts on the inside: academics reinventing themselves online. International Journal of Lifelong Education, 34(2), 194-210. 
*Cotterall, S. (2015). The rich get richer: international doctoral candidates and scholarly identity. Innovations in Education and Teaching International, 52 (4) 360-370.

Creswell, J. W., \& Poth, C. N. (2017). Qualitative inquiry and research design: choosing among five approaches (Fourth edition). Sage publications.

*Dison, A. (2004). "Finding her own academic self": research capacity development and identity formation. Perspectives in Education, 22(1), 83-98.

Elliott, J. (2005). Using narrative in social research: qualitative and quantitative approaches. London: Sage.

Epitropaki, O., Kark, R., Mainemelis, C., \& Lord, R. G. (2017). Leadership and followership identity processes: a multilevel review. The Leadership Quarterly, 28(1), 104-129.

*Gardner, A., \& Willey, K. (2018). Academic identity reconstruction: the transition of engineering academics to engineering education researchers. Studies in Higher Education, 43(2), 234-250.

González, M. A., Castelló, M., \& Monereo, C. (2014). The identity of the nursing academic: between education and research. Texto \& Contexto-Enfermagem, 23(2), 241-249.

Gough, D., Oliver, S. \& Thomas, J. (2017). An introduction to systematic reviews. Sage Publications.

Grant, M. J., \& Booth, A. (2009). A typology of reviews: an analysis of 14 review types and associated methodologies. Health Information and Libraries Journal, 26(2), 91-108.

Green, B. (2005). Unfinished business: subjectivity and supervision. Higher Education Research and Development, 24(2), 151-163.

*Guerin, C. (2013). Rhizomatic research cultures, writing groups and academic researcher identities. International Journal of Doctoral Studies, 8, 137-150.

*Gunasekara, C. (2007). Pivoting the centre: reflections on undertaking qualitative interviewing in academia. Qualitative Research, 7(4), 461-475.

Hammack, P. L. (2015). Theoretical foundations of identity. In K. C. McLean \& M. U. Syed (Eds.), The Oxford handbook of identity development (pp. 11-30). USA: Oxford University Press.

*Heinrich, K. T. (2005). Halfway between receiving and giving: a relational analysis of doctorate-prepared nurse-scholars' first 5 years after graduation. Journal of Professional Nursing, 21(5), 303-313.

Hermans, H. J. (2001). The dialogical self: toward a theory of personal and cultural positioning. Culture \& Psychology, 7(3), 243-281.

Hermans, H. J., \& Dimaggio, G. (2007). Self, identity, and globalization in times of uncertainty: a dialogical analysis. Review of General Psychology, 11(1), 31-61.

*Hökkä, P., Eteläpelto, A., \& Rasku-Puttonen, H. (2012). The professional agency of teacher educators amid academic discourses. Journal of Education for Teaching, 38(1), 83-102.

Holck, L., Muhr, S. L., \& Villesèche, F. (2016). Identity, diversity and diversity management: on theoretical connections, assumptions and implications for practice. Equality, Diversity and Inclusion: An International Journal, 35(1), 48-64.

*Holley, K. A. (2015). Doctoral education and the development of an interdisciplinary identity. Innovations in education and teaching international, 52(6), 642-652.

Ibarra, H., \& Barbulescu, R. (2010). Identity as narrative: prevalence, effectiveness, and consequences of narrative identity work in macro work role transitions. Academy of Management Review, 35(1), 135-154.

Inouye, K. S., \& McAlpine, L. (2017). Developing scholarly identity: variation in agentive responses to supervisor feedback. Journal of University Teaching and Learning Practice, 14(2), 3.

Ivanič, R. (1998). Writing and identity. John Benjamins.

Kennedy, M. (2007). Defining a literature. Educational Researcher, 36(3), 139-147.

Klenowski, V., Ehrich, L., Kapitzke, C., \& Trigger, K. (2011). Building support for learning within a Doctor of Education programme. Teaching in Higher Education, 16(6), 681-693.

Knez, I. (2016). Toward a model of work-related self: a narrative review. Frontiers in Psychology, 7(MAR).

Kuhn, T. S. (1962). The structure of scientific revolutions. Chicago: University of Chicago.

Lave, J., \& Wenger, E. (1991). Situated learning: Legitimate peripheral participation. Cambridge university press.

*Lassig, C. J., Dillon, L. H., \& Diezmann, C. M. (2013). Student or scholar? Transforming identities through a research writing group. Studies in Continuing Education, 35(3), 299-314.

*Leibowitz, B., Ndebele, C., \& Winberg, C. (2014). 'It's an amazing learning curve to be part of the project': exploring academic identity in collaborative research. Studies in Higher Education, 39(7), 1256-1269.

MacNaughton, G., \& Rolfe, S. A., \& I. Siraj-Blatchford. (2001). The research process. Doing early childhood research: international perspectives on theory and practice, 12-30.

*McAlpine, L. \& Amundsen, C. (2009). Identity and agency: pleasures and collegiality among the challenges of the doctoral journey. Studies in Continuing Education, (31) 2, 109-125.

*McAlpine, L., \& Lucas, L. (2011). Different places, different specialisms: similar questions of doctoral identities under construction. Teaching in Higher Education, 16(6), 695-706. 
*McAlpine, L., Amundsen, C., \& Turner, G. (2013). Constructing post-PhD careers: negotiating opportunities and personal goals. International Journal for Researcher Development, 4(1), 39-54.

*McAlpine, L., Amundsen, C., \& Turner, G. (2014). Identity-trajectory: reframing early career academic experience. British Educational Research Journal, 40(6), 952-969.

*Merolla, D. M., \& Serpe, R. T. (2013). STEM enrichment programs and graduate school matriculation: the role of science identity salience. Social Psychology of Education, 16(4), 575-597.

* Mewburn, I. (2011). Troubling talk: assembling the PhD candidate. Studies in Continuing Education, 33(3), 321-332.

*Murakami-Ramalho, E., Militello, M., \& Piert, J. (2013). A view from within: how doctoral students in educational administration develop research knowledge and identity. Studies in Higher Education, 38(2), 256-271.

Neuman, W. L. (2000). The meanings of methodology. Social research methods, 60, 87.

Palmer, R. J., Hunt, A. N., Neal, M., \& Wuetherick, B. (2015). Mentoring, undergraduate research, and identity development: a conceptual review and research agenda. Mentoring \& Tutoring: Partnership in Learning, 23(5), 411-426.

Pifer, M., \& Baker, V. (2013). Identity as a theoretical construct in research about academic careers. In Theory and method in higher education research (pp. 115-132). Emerald Group Publishing Limited.

*Pifer, M. J., \& Baker, V. L. (2016). Professional, personal, and relational: exploring the salience of identity in academic careers. Identity, 16(3), 190-205.

*Rayner, S., Lord, J., Parr, E., \& Sharkey, R. (2015). 'Why has my world become more confusing than it used to be? 'Professional doctoral students reflect on the development of their identity. Management in Education, 29(4), 158-163.

*Remich, R., Naffziger-Hirsch, M. E., Gazley, J. L., \& McGee, R. (2016). Scientific growth and identity development during a post-baccalaureate program: results from a multisite qualitative study. CBE-Life Sciences Education, 15(3), ar25.

Ricoeur, P. (1991). Narrative identity. Philosophy today

*Rockinson-Szapkiw, A. J., Spaulding, L. S., \& Lunde, R. (2017). Women in distance doctoral programs: how they negotiate their identities as mothers, professionals, and academics in order to persist. International Journal of Doctoral Studies, 12(7), 50-72.

*Schulze, S. (2014). Finding the academic self: identity development of academics as doctoral students. Koers, 79(1), 1-8.

*Smith, C., \& Boyd, P. (2012). Becoming an academic: the reconstruction of identity by recently appointed lecturers in nursing, midwifery and the allied health professions. Innovations in Education and Teaching International, 49(1), 63-72.

Sousa, F. J. (2010). Meta-theories in research: positivism, postmodernism, and critical realism. In Organizational culture, business-to-business relationships, and interfirm networks (pp. 455-503). Emerald Group Publishing Limited. Available at SSRN: https://papers.ssrn.com/sol3/papers.cfm?abstract_id=1594098 Accessed 20 Jun 2020

Strauss, A. L. (2017). Mirrors and masks: the search for identity. Routledge.

SwafS, Horizon 2020; https://ec.europa.eu/programmes/horizon2020/en/h2020-section/science-and-society

*Thompson, C., Morton, J., \& Storch, N. (2016). Becoming an applied linguist. Australian review of applied linguistics, 39(2), 139-157.

Trede, F., Macklin, R., \& Bridges, D. (2012). Professional identity development: a review of the higher education literature. Studies in Higher Education, 37(3), 365-384.

Van Lankveld, T., Schoonenboom, J., Volman, M., Croiset, G., \& Beishuizen, J. (2017). Developing a teacher identity in the university context: a systematic review of the literature. Higher Education Research and Development, 36(2), 325-342.

van Leeuwen, T. (2013). Bibliometric research evaluations, Web of Science and the Social Sciences and Humanities: a problematic relationship? Bibliometrie - Prxis und Forschung, Band 2. https://doi. org/10.5283/bpf.173

Vandenberghe, F. (2007). Structure, agency and the internal conversation. Revue du Mauss permanente, 1-8. Retrieved from http://www.journaldumauss.net Accessed 20 Jun 2020

*Wegener, C., Meier, N., \& Ingerslev, K. (2016). Borrowing brainpower-sharing insecurities. Lessons learned from a doctoral peer-writing group. Studies in Higher Education, 41(6), 1092-1105.

Wenger, E. (1999). Communities of practice: learning, meaning, and identity. Cambridge University press.

*Zambo, D., Buss, R. R., \& Zambo, R. (2015). Uncovering the identities of students and graduates in a CPEDinfluenced EdD program. Studies in Higher Education, 40(2), 233-252.

Zucchermaglio, C., \& Talamo, A. (2000). The social construction of work times: negotiated time and expected time. Time \& Society, 9(2-3), 205-222. 
Publisher's note Springer Nature remains neutral with regard to jurisdictional claims in published maps and institutional affiliations.

\section{Affiliations}

\section{Montserrat Castelló ${ }^{1,2} \cdot$ Lynn McAlpine ${ }^{3,4} \cdot$ Anna Sala-Bubaré $^{1} \cdot$ Kelsey Inouye $^{3}$. Isabelle Skakni ${ }^{5}$}

Lynn McAlpine

lynn.mcalpine@learning.ox.ac.uk; lynn.mcalpine@mcgill.ca

Anna Sala-Bubaré

annasb4@blanquerna.url.edu

Kelsey Inouye

kelsey.inouye@linacre.ox.ac.uk

Isabelle Skakni

i.skakni@lancaster.ac.uk

1 Facultat de Psicologia, Ciències de l'Educació i de l'Esport Blanquerna, Universitat Ramon Llull, C/ Cister 34, 08022 Barcelona, Spain

2 Graduate School of Psychology, Blanquerna, Ramon Llull University, C/ Cister 34, 08022 Barcelona, Spain

3 Department of Education, University of Oxford, Oxford, UK

4 Faculty of Education, McGill University, Montreal, Canada

5 Department of Educational Research, Lancaster University, Lancashire, UK 